\title{
Recognition and Reconstruction of Developable Surfaces from Point Clouds
}

\author{
Martin Peternell \\ Vienna University of Technology \\ Institute of Discrete Mathematics and Geometry \\ Wiedner Hauptstr. 8-10 \\ A-1040 Vienna, Austria \\ martin@geometrie.tuwien.ac.at
}

\begin{abstract}
Given a cloud $P$ of data points, we present techniques and algorithms to decide whether $P$ could be measurements of a developable surface and to reconstruct the original shape of the surface. We will use the dual approach to developable surfaces, which says that a developable surface can be considered as envelope of a one-parameter family of planes. A classification will be given which detects cones, cylinders, and developable surfaces of constant slope. Finally we discuss the problems occurring in this context.
\end{abstract}

\section{Introduction}

Given a cloud of data points $\mathbf{p}_{i}, i=1, \ldots, N$ in $\mathbb{R}^{3}$, we want to decide whether $\mathbf{p}_{i}$ are measurements of a developable surface. If this is the case, we will describe how to find a developable surface which approximates the data points $\mathbf{p}_{i}$. The problem sounds simpler as it is in fact. One would like to have a fully automatic procedure but the experience shows that there are certain limitations concerning the regularity of the approximation, see Section 5.5.

Points and vectors in $\mathbb{R}^{3}$ or $\mathbb{R}^{4}$ are denoted by boldface letters, $\mathbf{p}, \mathbf{v}$. Planes and lines are displayed as italic capital letters, $T, L$. We use Cartesian coordinates in $\mathbb{R}^{3}$ with axes $x, y$ and $z$. In $\mathbb{R}^{4}$ the axes of the Cartesian coordinate system are denoted by $u_{1}, \ldots, u_{4}$.

A ruled surface carries a one parameter family of straight lines $L$. The surface is in particular a developable surface $D$ if all surface points along a fixed line have the same tangent plane (with exception of a possibly occurring singular point). The general parametrization of a ruled surface is

$$
\mathbf{x}(u, v)=\mathbf{c}(u)+v \mathbf{e}(u)
$$

where $\mathbf{c}(u)$ is called directrix curve and $\mathbf{e}(u)$ is a vector field along $\mathbf{c}(u)$, which describes the generating straight lines. The normal vectors of the ruled surface $\mathbf{x}(u, v)$ are

$$
\mathbf{n}(u, v)=\dot{\mathbf{c}}(u) \times \mathbf{e}(u)+v \dot{\mathbf{e}}(u) \times \mathbf{e}(u) .
$$

For fixed $u=u_{0}$, the normal vectors are linear combinations of the vectors $\dot{\mathbf{c}}\left(u_{0}\right) \times \mathbf{e}\left(u_{0}\right)$ and $\dot{\mathbf{e}}\left(u_{0}\right) \times \mathbf{e}\left(u_{0}\right)$. If $\mathbf{x}(u, v)$ is a developable surface, the two components $\dot{\mathbf{c}} \times \mathbf{e}$ and $\dot{\mathbf{e}} \times \mathbf{e}$ are linearly dependent. This gives the following characterization: The parametrization $\mathbf{x}(u, v)$ describes a developable surface exactly if the condition

$$
\operatorname{det}(\dot{\mathbf{c}}, \mathbf{e}, \dot{\mathbf{e}})=0
$$

is identically satisfied. This says that the normals along a generating line are all parallel which implies that the tangent plane $T(u)$ along a generating line $L$ is fixed. There exists a unique singular point $\mathbf{s}(\mathrm{u})$ at each generating line $L(u)$, and it is determined by the parameter value

$$
v_{s}=-\frac{(\dot{\mathbf{c}} \times \mathbf{e}) \cdot(\dot{\mathbf{e}} \times \mathbf{e})}{(\dot{\mathbf{e}} \times \mathbf{e})^{2}} .
$$

If $\mathbf{e}$ and $\dot{\mathbf{e}}$ are linearly dependent, the singular point $\mathbf{s}$ is at infinity. In Euclidean space $\mathbb{R}^{3}$ there exist three different classes of developable surfaces:

1. Cylinders: the singular curve degenerates to a single point at infinity.

2. Cones: the singular curve degenerates to a single proper point, which is called vertex.

3. General Case: these surfaces consist of the tangent lines of a regular space curve $\mathbf{s}(u)$, which is the singular curve of the surface.

In all three cases the surface can be generated as envelope of its one parameter family of tangent planes. A cylinder of revolution is obtained by rotating a plane around an axis which is parallel to that plane. A cone of revolution is obtained by rotating a plane around a general axis, but which is not perpendicular to that plane. Further, it is known that smooth developable surfaces can be characterized by vanishing Gaussian curvature. 


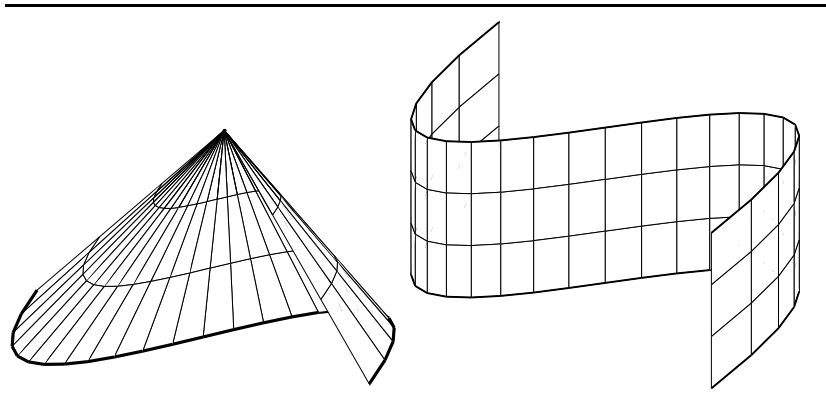

Figure 1. General cylinder and cone

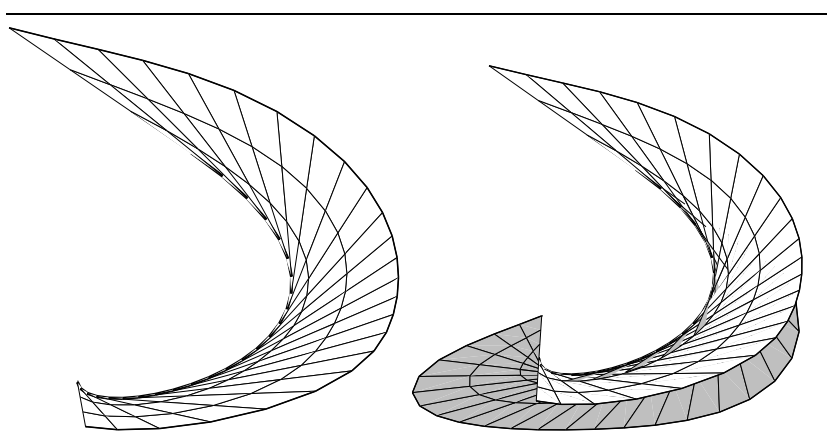

Figure 2. General developable surface: one and two sheets of the tangent surface of a space curve

\subsection{Contribution of the article}

There is quite a lot of literature on modelling with developable surfaces, see for instance [1, 2, 3, 7, 12, 15]. Bspline representations and the dual representation are well known and have been used for interpolation and approximation tasks.

The contribution of this paper is the focus on the reconstruction of developable surfaces from scattered data by the use of estimated tangent planes for the data points. The approximation is performed in the space of planes with respect to a meaningful chosen metric. In addition, we use a data structure (tesselation) in the space of planes, which guarantees quick data access. The proposed algorithm can also be applied to approximation of arbitrary (nearly developable) surfaces by developable surfaces. The test implementations have been performed in Matlab and the data have been generated by scanning models of developable surfaces with an optical laser scanner.

The article is organized as follows: Section 2 contains the dual approach together with a discussion of the Blaschke image curves of developable surfaces. Section 3 tells about a classification of developable surfaces with help of the Blaschke image. Section 4 discusses the recognition of these surfaces in point clouds. Section 5 gives two algorithms to reconstruct these surfaces from data points. Finally, we present some examples and discuss problems and possible solutions.

\section{The dual approach}

Let $T(u)$ be a one-parameter family of planes

$$
T(u): n_{4}(u)+n_{1}(u) x+n_{2}(u) y+n_{3}(u) z=0
$$

with arbitrary functions $n_{i}, i=1, \ldots, 4$. The vector $\mathbf{n}(u)=$ $\left(n_{1}, n_{2}, n_{3}\right)(u)$ is a normal vector of $T(u)$. The developable envelope surface $D$ of $T(u)$ contains generating lines $L(u)$ which are obtained by intersecting $T(u) \cap \dot{T}(u)$, where $\dot{T}(u)$ denotes the derivative with respect to $u$. The generating lines themselves envelope the singular curve $\mathbf{s}(u)$ which is obtained by the intersection $T(u) \cap \dot{T}(u) \cap \ddot{T}(u)$.

The functions $n_{i}$ of $T(u)$ are not uniquely defined, but any $\rho n_{1}, \rho n_{2}, \rho n_{3}, \rho n_{4}$ with a smooth function $\rho(u)$ describes the same family of planes $T(u)$. So, we can assume that $T(u)$ is given in Hesse normal form which satisfies the condition

$$
n_{1}^{2}+n_{2}^{2}+n_{3}^{2}=1 .
$$

This is the normalization of the normal vector $\mathbf{n}(u)$. We consider $n_{1}, n_{2}, n_{3}, n_{4}$ as coordinate functions in a space $\mathbb{R}^{4}$, with coordinate axes $u_{1}, u_{2}, u_{3}, u_{4}$. The normalization condition is

$$
B: u_{1}^{2}+u_{2}^{2}+u_{3}^{2}=1,
$$

which describes a hypersurface in $\mathbb{R}^{4}$.

\subsection{The Blaschke mapping}

The coefficients $n_{i}$ of planes $E: n_{4}+n_{1} x+n_{2} y+$ $n_{3} z=0$ can be interpreted as coordinates of points $E^{*}=$ $\left(n_{1}, n_{2}, n_{3}, n_{4}\right)$ on $B$, if we take the condition (5) into account. The vectors $\left(n_{1}, \ldots, n_{4}\right)$ and $\left(-n_{1}, \ldots,-n_{4}\right)$ define the same carrier plane, but their unit normals $\mathbf{n}$ and $-\mathbf{n}$ have opposite orientations. Thus, each carrier plane carries two different oriented planes and orientation is defined with respect to the oriented unit normal $\mathbf{n}$.

The hypersurface $B$ is a quadratic cylinder and the intersections of $B$ with hyperplanes $u_{4}=$ const. are spheres with radius 1. In particular, the intersection with $u_{4}=0$ is the unit sphere in $\mathbb{R}^{3}$ (if $\mathbb{R}^{3}$ is embedded in $\mathbb{R}^{4}$ as hyperplane $u_{4}=0$ ).

Let $b$ be the mapping from the set of oriented planes $E$ to their image points $b(E) \in B$, defined by

$b: E: n_{4}+n_{1} x+n_{2} y+n_{3} z=0 \mapsto b(E)=\left(n_{1}, n_{2}, n_{3}, n_{4}\right)$.

We call it Blaschke mapping and $B$ is called Blaschke cylinder. $B$ is the image of the set of oriented planes $E \in \mathbb{R}^{3}$. 
Any developable surface $D$ can be considered as oneparameter family of tangent planes $T(u)$, thus it can be interpreted as curve $b(T(u))=b(D)$ on $B \subset \mathbb{R}^{4}$. Conversely, any differentiable curve $C(u) \subset B$ which is not a generating line of $B$, can be considered as Blaschke image $b(T(u))$ of a one parameter family of planes $T(u)$, whose envelope is a developable surface $D$ (including the degenerate case of a pencil of planes). Thus, $C(u)$ can be interpreted as Blaschke image of $D$. By the way, the points of a generating line on $B$ correspond to a family of parallel planes in $\mathbb{R}^{3}$.

\subsection{Tangent planes of a sphere}

We have to investigate the Blaschke images of the tangent planes $T$ of a sphere $S$ in $\mathbb{R}^{3}$ because these results will be used for the classification of developable surfaces in Section 3. Let $S$ be given by the equation

$$
S:\left(x-m_{1}\right)^{2}+\left(y-m_{2}\right)^{2}+\left(z-m_{3}\right)^{2}-r^{2}=0 .
$$

The sphere can be considered as oriented surface with help of oriented unit normals. Equivalently, we can orient spheres by considering signed radii, thus the spheres with center $\mathbf{m}=\left(m_{1}, m_{2}, m_{3}\right)$ and radii $\pm r$ are different.

An oriented plane $T: n_{4}+n_{1} x+n_{2} y+n_{3} z=0$ is tangent to the oriented sphere $S$, exactly if

$$
n_{4}+n_{1} m_{1}+n_{2} m_{2}+n_{3} m_{3}-r=0 .
$$

This says that the Blaschke images $b(T)$ of tangent planes $T$ of $S$ satisfy a linear equation of the form

$$
-r+u_{1} m_{1}+u_{2} m_{2}+u_{3} m_{3}+u_{4}=0 .
$$

Conversely, if points $\mathbf{q}=\left(q_{1}, q_{2}, q_{3}, q_{4}\right) \in B$ satisfy a linear relation

$$
H: a_{0}+u_{1} a_{1}+u_{2} a_{2}+u_{3} a_{3}+u_{4} a_{4}=0,
$$

$\mathbf{q}=b(T)$ are Blaschke images of planes $T$ which are tangent to a sphere in case $a_{4} \neq 0$. Center and radius are determined by

$$
\mathbf{m}=\frac{1}{a_{4}}\left(a_{1}, a_{2}, a_{3}\right), \quad r=\frac{-a_{0}}{a_{4}} .
$$

If $a_{0}=0$, the planes $b(T)$ pass through the fixed point $\mathbf{m}$. If $a_{4}=0$, the planes $T$ form a constant angle with the direction vector $\mathbf{a}=\left(a_{1}, a_{2}, a_{3}\right)$ because of $\mathbf{a} \cdot \mathbf{n}=-a_{0}$, with $\mathbf{n}=\left(u_{1}, u_{2}, u_{3}\right)$.

\section{The classification of developable surfaces}

We will discuss how cylinders and cones $D$ and other special developable surfaces can be characterized by their Blaschke image $b(D)$.
Cylinder: The surface $D$ is a cylinder if all its tangent planes $T(u)$ are parallel to a vector a. Thus, the normal vectors $\mathbf{n}(u)$ are perpendicular to a. This implies that the image points $b(T)$ of the tangent planes $T$ of $D$ satisfy $\mathbf{n} \cdot \mathbf{a}=n_{1} a_{1}+n_{2} a_{2}+n_{3} a_{3}=0$. Thus, the curve $b(T(u))$ is contained in the three-space

$$
a_{1} u_{1}+a_{2} u_{2}+a_{3} u_{3}=0 .
$$

Cone: The surface $D$ is a cone if all its tangent planes $T(u)$ pass through a fixed point $\mathbf{p}=\left(p_{1}, p_{2}, p_{3}\right)$. This incidence is expressed by $p_{1} n_{1}+p_{2} n_{2}+p_{3} n_{3}+n_{4}=0$. Thus, the Blaschke image curve $b(T(u))=b(D)$ is contained in the three-space

$$
p_{1} u_{1}+p_{2} u_{2}+p_{3} u_{3}+u_{4}=0 \text {. }
$$

There exist other special types of developable surfaces. Two of them will be mentioned here.

The surface $D$ is a developable of constant slope, if its normal vectors $\mathbf{n}$ form a constant angle $\phi$ with a fixed direction vector a. Assuming that $\|\mathbf{a}\|=1$, we get $\cos (\phi)=$ $\mathbf{a} \cdot \mathbf{n}=\gamma=$ const. This implies that the Blaschke images of the tangent planes of $D$ are contained in the three-space

$$
-\gamma+a_{1} u_{1}+a_{2} u_{2}+a_{3} u_{3}=0 .
$$

The developable surface $D$ is tangent to a sphere with center $\mathbf{m}$ and radius $r$, if the tangent planes $T(u)$ of $D$ satisfy $n_{4}+n_{1} m_{1}+n_{2} m_{2}+n_{3} m_{3}-r=0$. Thus, the Blaschke image curve $b(D)$ is contained in the three-space

$$
-r+u_{1} m_{1}+u_{2} m_{2}+u_{3} m_{3}+u_{4}=0 .
$$

It is of particular interest if a developable $D$ is a cone or cylinder of revolution. These two cases are discussed in the next section.

\subsection{Cones and cylinders of revolution}

Let $D$ be a cylinder of revolution with axis $A$ and radius $r$. The tangent planes $T$ of $D$ are tangent to all spheres of radius $r$, whose centers vary on $A$. We consider two spheres

$$
S_{1}:(\mathbf{x}-\mathbf{p})^{2}-r^{2}=0, \quad S_{2}:(\mathbf{x}-\mathbf{q})^{2}-r^{2}=0
$$

with equal radii. The Blaschke images $b(T)$ satisfy the relations

$$
\begin{aligned}
& H_{1}: \quad-r+u_{1} p_{1}+u_{2} p_{2}+u_{3} p_{3}+u_{4}=0, \\
& H_{2}: \quad-r+u_{1} q_{1}+u_{2} q_{2}+u_{3} q_{3}+u_{4}=0,
\end{aligned}
$$

which implies that the Blaschke image curve $b(D)$ is a planar curve (conic) $B \cap P$, where $P$ is the plane determined by (14). 
Cones of revolution $D$ can be obtained as envelopes of the common tangent planes of two spheres $S_{1}, S_{2}$ with different radii $r \neq s$. Thus, $b(D)$ is a conic contained in the plane $P$ defined by

$$
\begin{array}{lll}
H_{1}: & -r+u_{1} p_{1}+u_{2} p_{2}+u_{3} p_{3}+u_{4}=0 \\
H_{2}: & -s+u_{1} q_{1}+u_{2} q_{2}+u_{3} q_{3}+u_{4}=0 .
\end{array}
$$

Conversely, if the Blaschke image $b(D)$ of a developable surface is a planar curve, contained in a plane $P$, how can we decide whether $D$ is a cone or cylinder of revolution? Let $b(D)=b(T(u))$ be a planar curve $\subset P$ and let $P$ be given as intersection of two three-spaces $H_{1}, H_{2}$, with

$$
H_{i}: h_{i 0}+h_{i 1} u_{1}+h_{i 2} u_{2}+h_{i 3} u_{3}+h_{i 4} u_{4}=0 \text {. }
$$

We have already found in Section 2.2 that if the Blaschke image $b(T)$ is contained in a three-space $H_{i}$, the corresponding tangent plane $T$ is tangent to a sphere, or is passing through a point, or encloses a fixed angle with a fixed direction. This property has to hold with respect to $H_{1}$ and $H_{2}$. If we exclude the degenerate case $h_{14}=h_{24}=0$, we can assume that $P$ is determined by two three-spaces $H_{1}, H_{2}$ of the form (14) or (15).

1. At first, the plane $P$ is defined by three-spaces $H_{1}, H_{2}$ given by equations (14). Then, the developable surface $D$ is a cylinder of revolution. By subtracting the equations (14) we find that the normal vectors $\mathbf{n}$ of $T(u)$ have to satisfy

$$
\mathbf{n} \cdot(\mathbf{p}-\mathbf{q})=0 \text {. }
$$

Thus, the axis $A$ of $D$ is given by $\mathbf{a}=\mathbf{p}-\mathbf{q}$ and $D$ 's radius equals $r$.

2. At second, the plane $P$ is defined by three-spaces $H_{1}, H_{2}$ given by equations (15). The pencil of threespaces $\lambda H_{1}+\mu H_{2}$ contains a unique three-space $H$, passing through the origin, whose equation is

$$
H: \sum_{i=1}^{3} u_{i}\left(s p_{i}-r q_{i}\right)+u_{4}(s-r)=0 .
$$

Thus, the tangent planes of the developable surface $D$ are passing through a fixed point, corresponding to $H$, and $D$ is a cone of revolution. Its $\mathbf{v}$ vertex is

$$
\mathbf{v}=\frac{1}{s-r}(s \mathbf{p}-r \mathbf{q}) \text {. }
$$

The axis $A$ of $D$ is given by $\mathbf{a}=\mathbf{p}-\mathbf{q}$ and the inclination angle $\phi$ between the axis $A$ and the tangent planes $T(u)$ is

$$
\sin \phi=\frac{|s-r|}{\|\mathbf{q}-\mathbf{p}\|}
$$

\section{Recognition of Developable Surfaces}

Given a cloud of data points $\mathbf{p}_{i}$, this section discusses the recognition of developable surfaces and the classification, which type of surface we are dealing with. The algorithm contains the following steps:

1. Estimation of tangent planes $T_{i}$ for all data points $\mathbf{p}_{i}$.

2. Computing the Blaschke images $b\left(T_{i}\right)$ of $T_{i}$.

3. Analysis of the structure of the set $\mathcal{T}$ of image points $b\left(T_{i}\right)$.

4. If $\mathcal{T}$ is curve-like, classification of the developable surface, which is close to $\mathbf{p}_{i}$.

\subsection{Estimation of tangent planes}

Given the data points $\mathbf{p}_{i}$ with Cartesian coordinates $x_{i}, y_{i}, z_{i}$ in $\mathbb{R}^{3}$, we assume that a triangulation of the data points is already computed. So, we are given in addition a list of triangles $t_{j}=\left(j_{1}, j_{2}, j_{3}\right)$, consisting of triples of integer values, which are references to the point list $\mathbf{p}_{i}$. This gives topological information for the point cloud, and we are able to define neighboring points $\mathbf{q}_{k}$ for all data points $\mathbf{p}_{i}$. In addition we assume that the data points $\mathbf{p}_{i}$ represent a surface with orientation which is reflected by oriented triangles $t_{j}$.

For each data point $\mathbf{p}_{i}$, we want to estimate a tangent plane $T_{i}$. So, let $\mathbf{p}$ be a fixed point and let $\mathbf{q}_{k}$ be $\mathbf{p}^{\prime} s$ neighbors. The estimated tangent plane $T$ at $\mathbf{p}$ shall be a plane best fitting the data points $\mathbf{q}_{k} . T$ can be computed as minimizer (in the $l_{1}$ or $l_{2}$-sense) of the vector of distances $d\left(\mathbf{q}_{k}, T\right)$ between the data points $\mathbf{q}_{k}$ and the plane $T$. This leads to a set of estimated tangent planes $T_{i}: d_{i}+a_{i} x+b_{i} y+c_{i} z=0$, corresponding to the set of data points $\mathbf{p}_{i}$ and we use the normalization condition $a_{i}^{2}+b_{i}^{2}+c_{i}^{2}=1$. For more information concerning reverse engineering, see the survey [17].

\subsection{Blaschke image of estimated tangent planes}

The Blaschke images $b\left(T_{i}\right)=\left(a_{i}, b_{i}, c_{i}, d_{i}\right)$ of the estimated tangent planes $T_{i}$ form a point cloud on the Blaschke cylinder $B: u_{1}^{2}+u_{2}^{2}+u_{3}^{2}=1$. The original surface with measurement points $\mathbf{p}_{i}$ is a developable surface, if the image points $b\left(T_{i}\right)$ form a curve-like region on $B$. To check the property curve-like, it is necessary to define neighborhoods on $B$. Another possibility, which is similar to this, is the definition of a distance $d\left(T_{1}, T_{2}\right)$ between two planes $T_{1}, T_{2}$. For this, we have to make some assumptions on our data points $\mathbf{p}_{i}$.

We apply a uniform scaling of the data points in a way that all coordinates $x_{i}, y_{i}, z_{i}$ are $\leq c=1 / \sqrt{3}$. Thus, the object is contained in a cube, bounded by the planes 
$x= \pm c, y= \pm c, z= \pm c$. This implies that the maximum distance of a data point $\mathbf{p}_{i}$ and the origin is 1 . In addition, the maximum distance $d\left(O, T_{i}\right)$ of an estimated tangent plane $T_{i}$ to the origin is 1 . Since $d\left(O, T_{i}\right)=d_{i}$, the fourth coordinates $d_{i}$ of the Blaschke images $b\left(T_{i}\right)$ are bounded by \pm 1 . We define the squared distances between two planes $T_{1}, T_{2}$ according to the canonical Euclidean distance in $\mathbb{R}^{4}$, by

$d\left(T_{1}, T_{2}\right)^{2}=\left(a_{1}-a_{2}\right)^{2}+\left(b_{1}-b_{2}\right)^{2}+\left(c_{1}-c_{2}\right)^{2}+\left(d_{1}-d_{2}\right)^{2}$.

To study this distance function in $\mathbb{R}^{3}$, we consider a fixed plane $M$ and all planes $X$ with $d(M, X)<r$. The boundary of the region for which $d(X, M)=r$ holds, is illustrated in the 2D-Figure 3. It shows three 'planes' $M_{i}, i=0,1,2$ with different distances to the origin and the boundaries for $r=0.25$. The tangents $X$ of the boundaries are 'planes' satisfying $d\left(X, M_{i}\right)=r$. The extremal perpendicular distance of $X$ and $M$ is $r$ and the extremal turning angle between $X$ and $M$ is indicated by the dotted asymptotic lines of the boundaries.

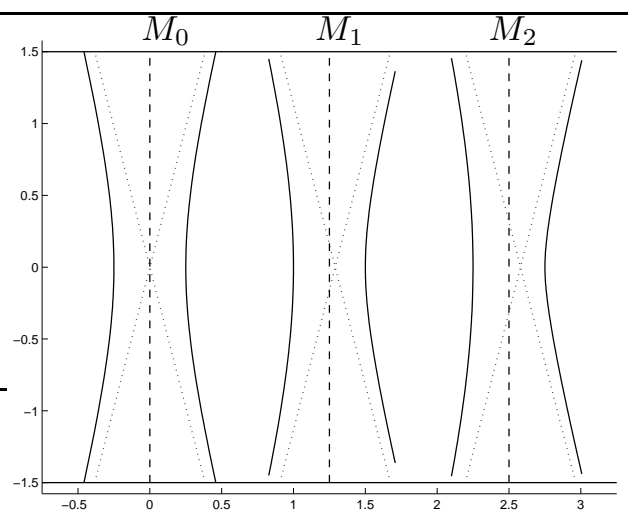

Figure 3. Regions for $d\left(X, M_{i}\right)<r$.

For practical computations on $B$ we use a cell decomposition of $B$ to define neighborhoods of image points $b(T)$ of (estimated) tangent planes $T$.

\subsection{A cell decomposition of the Blaschke cylinder}

We recall that $B$ 's equation is $u_{1}^{2}+u_{2}^{2}+u_{3}^{2}=1$. Any cross section with planes $u_{4}=$ const. is a copy of the unit sphere $S^{2}$ in $\mathbb{R}^{3}$. So, we start with a decomposition of $S^{2}$ and lift it to $B$.

A tessellation of $S^{2}$ can be based on the net of a regular icosahedron. Let $v_{i}, i=1, \ldots, 12$, with $\left\|v_{i}\right\|=1$, be vertices of a regular icosahedron. The vertices $v_{i}$ form twenty triangles $t_{j}$ and thirty edges. All edges have same arc length. This icosahedral net is subdivided by computing the midpoints of all edges (geodesic circles). Any trian- gle $t_{j}$ is subdivided into four new triangles. The inner triangle has equal edge lengths, the outer three have not, but the lengths of the edges to not vary too much.

Figure 4 shows an icosahedron and one refinement step. For simplicity only the polyhedra are drawn and not the geodesic nets on the unit sphere. The final tesselation of the unit sphere is displayed in Figures 5 and 7.

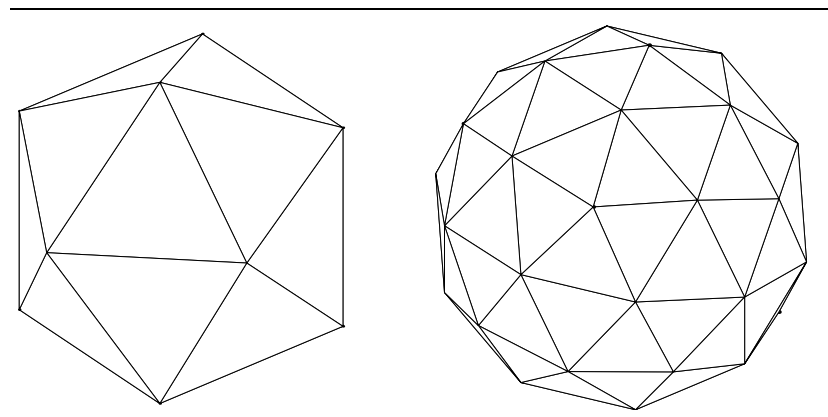

Figure 4. Icosahedron and refinement step

The cell decomposition of the Blaschke cylinder is now computed in the following way: Since we measure distances according to (17), the height of a prismatic cell have to be approximately equal to the edge length of a triangle. As we have mentioned earlier, the values of the fourth coordinate in $\mathbb{R}^{4}$ are bounded by \pm 1 . When each triangle of the tessellation is subdivided into four children, each interval of the fourth coordinate is divided into two subintervals. We start with 20 triangles, 12 vertices and 2 intervals in $u_{4}$-direction. The test-implementation uses the resolution after four subdivision steps with 1280 triangles, 642 vertices and 16 intervals in $u_{4}$-direction.

In addition to the vertices and cells on $B$ we store a list with adjacency information. Since each triangle on $S^{2}$ has eleven or twelve neighbors, a cell on $B$ has at most 38 adjacent cells.

Remark concerning the visualization: It is easy to visualize the spherical image (first three coordinates) on $S^{2}$, but it is hard to visualize the Blaschke image on $B$. We confine ourself to plot the spherical image on $S^{2}$, and if necessary, we add the fourth coordinate in a separate figure. This seems to be an appropriate visualization of the geometry on the Blaschke cylinder.

\subsection{Analysis of the Blaschke image}

Having computed estimates $T_{i}$ of the tangent planes of the data points and their images $b\left(T_{i}\right)$, we can check whether the Blaschke image of the considered surface is curve-like, see [17] for more information on reverse engineering and how to decide if a point cloud is curve-like. According to Section 4.3, the interesting part of the Blaschke 
cylinder $B$ is covered by $1280 \times 16$ cells $C_{k}$. We compute the memberships of image points $b\left(T_{i}\right)$ and cells $C_{k}$ and obtain a binary image on the cell structure $C$ of $B$.

1. If the data points $\mathbf{p}_{i}$ are contained in a single plane $P$, the image points $b\left(T_{i}\right)$ form a point-like cluster around $b(P)$.

2. If the data points $\mathbf{p}_{i}$ are contained in a developable surface, the image points $b\left(T_{i}\right)$ form a curve-like region in $B$, see Figure 5 .

3. If the data points $\mathbf{p}_{i}$ are contained in a doubly curved surface $S$, the image points $b\left(T_{i}\right)$ cover a two dimensional region on $B$.

In the following we assume that the surface under consideration is a smooth developable surface. Since the estimation of tangent planes gives bad results on the boundary of the surface patch and near measurement errors, there will be outliers in the Blaschke image. To find those, we search for cells $C_{k}$ carrying only a few image points. These cells and image points are not considered for the further computations. The result is referred to as cleaned Blaschke image. Figure 5 shows the original and the cleaned Blaschke image of a developable surface patch.

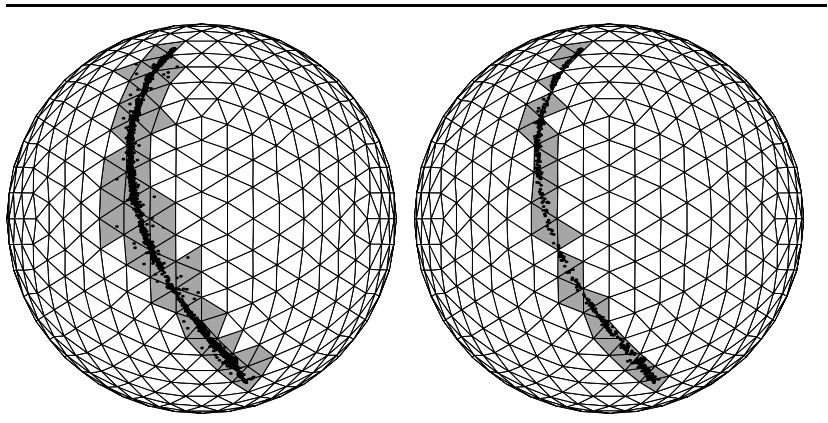

Figure 5. Original and cleaned Blaschke image of a developable surface

\subsection{Classifying the Blaschke image}

After having analyzed and cleaned the Blaschke image from outliers we are able to decide whether the given developable surface $D$ is a general cone or cylinder, a cone or cylinder of revolution, another special developable or a general developable surface.

So, let $T_{l}$ be the reliable planes of $D$ after the cleaning and let $b\left(T_{l}\right)$ be their Blaschke images. As we have worked out in Section 3 we can classify in the following way.

We compute the ellipsoid of inertia with respect to the data points $b\left(T_{l}\right)$ and obtain the axes vectors $\mathbf{h}_{i}$ of the ellipsoid as eigenvectors to corresponding eigenvalues $\lambda_{i}$. Let the eigenvalues be sorted by $\lambda_{1} \leq \lambda_{2} \leq \lambda_{3} \leq \lambda_{4}$. The smallest eigenvalue $\lambda_{1}$ determines the best fitting threespace $H_{1}$ of the point cloud $b\left(T_{l}\right)$ and $\mathbf{h}_{1}$ is its normal vector.

By investigating the magnitude of the eigenvalues $\lambda_{i}$ of the eigenvectors $\mathbf{h}_{i}$ and the coefficients of $H_{i}$ we can classify the type of surface $D$. Let $H_{i}$ be given by the equations

$$
H_{i}: h_{i 0}+h_{i 1} u_{1}+h_{i 2} u_{2}+h_{i 3} u_{3}+h_{i 4} u_{4}=0 .
$$

1. Two small eigenvalues $\lambda_{1}, \lambda_{2}$ and different coefficients $h_{10}, h_{20},\left(\left|h_{10}-h_{20}\right|>\varepsilon\right)$ : The surface $D$ can be well approximated by a cone of revolution, compare (15). The vertex and the inclination angle are computed according to Section 3.

2. Two small eigenvalues $\lambda_{1}, \lambda_{2}$ but nearly equal coefficients $h_{10}, h_{20},\left(\left|h_{10}-h_{20}\right| \leq \varepsilon\right)$ : The surface $D$ can be well approximated by a cylinder of revolution, compare (14). The axis and the radius are computed according to Section 3.

3. One small eigenvalue $\lambda_{1}$ and small coefficient $h_{10}$ (compare (11)): The surface $D$ is a general cone and its vertex is

$$
\mathbf{v}=\frac{1}{h_{14}}\left(h_{11}, h_{12}, h_{13}\right) .
$$

4. One small eigenvalue $\lambda_{1}$ and small coefficients $h_{10}$ and $h_{14}$ (compare (10)): The surface $D$ is a general cylinder and its axis is parallel to the vector

$$
\mathbf{a}=\left(h_{11}, h_{12}, h_{13}\right) .
$$

5. One small eigenvalue $\lambda_{1}$ and small coefficient $h_{14}$ (compare (12)): The surface $D$ is a developable of constant slope. The tangent planes of $D$ form a constant angle with respect to an axis. The angle and the axis are found according to formula (12). An example is displayed in Figure 6.

6. One small eigenvalue $\lambda_{1}$ characterizes a developable surface $D$ whose tangent planes $T_{i}$ are tangent to $a$ sphere (compare (13)). Its center and radius are

$$
\mathbf{m}=\frac{1}{h_{14}}\left(h_{11}, h_{12}, h_{13}\right), \quad r=\frac{-h_{10}}{h_{14}} .
$$

For this classification we need to fix a threshold $\varepsilon$, to decide what small means. This value depends on the accuracy of the measurement device, the number of data points per area unit and the accuracy of the object. Some experience is necessary to choose this value for particular applications.

Let $L$ be a generating line of a developable surface $D$ and let $T$ be the tangent plane along $L$. The Blaschke image of $(L, T)$ is a line element (point plus tangent line) on 


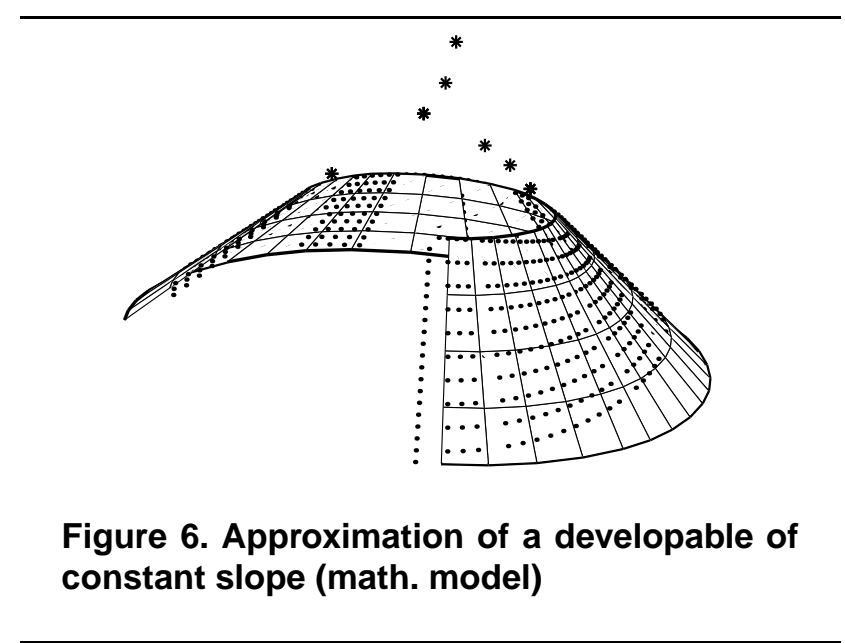

$B$. If $L$ is regular and not an inflection generator, then there exists a unique cone or cylinder of rotation $C$ which has second order contact with $D$ along $L$. The Blaschke image $b(C)$ is the unique osculating conic of $b(D)$ at $b(T)$.

This implies that a sufficiently small strip on a developable surface can always be well approximated by a cone or cylinder of rotation.

\section{Reconstruction of Developable Surfaces from Measurements}

So far, we have done some preparation steps for the reconstruction. In this section we describe how to define a one-parameter family of planes $E(t)$ best fitting the data points $\mathbf{p}_{i}$ or the estimated tangent planes $T_{i}$. In addition we will point to some problems, in particular the control of the singular curve of the approximation. First we point to some general demands on the surface $D$ to be approximated.

1. $D$ is a smooth surface not carrying singular points. It needs not be developable, but one can run the algorithm also for nearly developable surfaces (one small principal curvature).

2. The density of data points $\mathbf{p}_{i}$ has to be approximately the same everywhere.

3. The image $b\left(T_{i}\right)$ of the set of (estimated) tangent planes $T_{i}$ has to be a simple, curve-like region on the Blaschke cylinder which can be injectively parameterized over an interval.

According to the made assumptions, the reconstruction of a set of measurement point $\mathbf{p}_{i}$ of a developable surface $D$ can be divided into the following tasks:

1. Estimation of parameter values $t_{i}$ for image points $b\left(T_{i}\right)$ of estimated tangent planes $T_{i}$.

2. Approximation of points $b\left(T_{i}\right)$ plus parameter values $t_{i}$ by a curve $\mathbf{c}(t) \subset B$.
3. Computation of the one-parameter family of planes $E(t)$ in $\mathbb{R}^{3}$ and of the generating lines $L(t)$ of the developable $D^{*}$ which approximates measurements $\mathbf{p}_{i}$.

4. Computation of the boundary curves of $D^{*}$ with respect to the domain of interest in $\mathbb{R}^{3}$.

\subsection{Estimation of parameter values}

To simplify the approximation of points $b\left(T_{i}\right)$ by a curve $\mathbf{c}(t)$ later on, it is appropriate to estimate parameter values. There exist rather sophisticated methods, also for unorganized sets of data points, see [9]. Since we will use the cell structure on $B$, we propose the following algorithm. It uses the definition of a neighborhood $U_{0}$ of a data point $b\left(T_{0}\right)$. To define $U_{0}$, we estimate the width $w$ of the curve-like point cloud $b\left(T_{i}\right)$. The neighborhood $U_{0}$ is defined with respect to the cell structure on $B$ and its diameter has to be chosen significantly larger than the width $w$.

1. We start at an arbitrary data point $b\left(T_{0}\right)$ whose neighborhood is $U_{0}$. Let $b\left(T_{k}\right) \in U_{0}$ be the neighbors of $b\left(T_{0}\right)$. We compute the ellipsoid of inertia (principal axes and eigenvalues) for $b\left(T_{k}\right)$ and we obtain one significantly larger eigenvalue $\lambda_{1}$ and three smaller ones, due to the property of $U_{0}$. The eigenvector $\mathbf{v}_{1}$ to $\lambda_{1}$ is an estimate of the tangent vector at $b\left(T_{0}\right)$. By projecting the points $b\left(T_{k}\right)$ orthogonally onto the line $b\left(T_{0}\right)+\mu \mathbf{v}_{1}$ we can locally estimate parameter values

$$
t_{k}=\left[b\left(T_{k}\right)-b\left(T_{1}\right)\right] \cdot \mathbf{v}_{1}
$$

to data points $b\left(T_{k}\right) \in U_{0}$. We associate positive values $t_{k}$ to points $b\left(T_{k}\right)$ which lie in direction of $\mathbf{v}_{1}$ and negative values for points lying in direction $-\mathbf{v}_{1}$.

2. Now we perform a march in positive as well as in negative direction (with respect to the eigenvector $\mathbf{v}_{1}$ ) through the curve-like point cloud $b\left(T_{i}\right)$. For that we choose a new starting point $b\left(T_{+}\right) \in U_{0}$ for the positive direction with respect to $\mathbf{v}_{1}$. The point $b\left(T_{+}\right)$has to be close to the boundary of $U_{0}$ and in addition, its vertical distance from the line $b\left(T_{0}\right)+\mu \mathbf{v}_{1}$ shall be small. Its parameter value is denoted by $t_{+}$. The new starting point for the negative direction with respect to $\mathbf{v}_{1}$ is defined analogously. In the following we only describe the procedure for the positive direction with respect to $U_{0}$ and $\mathbf{v}_{1}$.

3. At each new starting point $b\left(T_{0}\right):=b\left(T_{+}\right)$we choose a neighborhood $U_{0}$ and determine neighboring points $b\left(T_{k}\right) \in U_{0}$. We compute an ellipsoid of inertia with respect to points $b\left(T_{k}\right)$ and estimate the tangent vector $\mathbf{v}_{1}$ at $b\left(T_{0}\right)$. This allows the local estimation of parameter values $t_{k}^{l o c}$ for the data points $b\left(T_{k}\right) \in U_{0}$. Since 
the starting point $b\left(T_{0}\right)$ already corresponds to the parameter value $t_{+}$, we associate values

$$
t_{k}=t_{+}+t_{k}^{l o c}
$$

to the data points $b\left(T_{k}\right)$. We choose a new starting point close to the boundary of $U_{0}$ and close to $b\left(T_{0}\right)+$ $\mu \mathbf{v}_{1}$ and repeat this step.

4. If the data points $b\left(T_{i}\right)$ form a closed curve, we repeat the last step until we have associated parameter values to all data points. Otherwise we have to perform the march also in negative direction, as already mentioned.

5. Later on, when having computed an approximating curve $\mathbf{c}(t)$ to the data $b\left(T_{i}\right)$, we can apply a parameter correction to improve the estimation, according to Hoschek, [6].

\subsection{Computing the approximating curve}

We are given data points $b\left(T_{i}\right)$ and parameter values $t_{i}$ and want to compute a curve $\mathbf{c}(t) \subset B$ best fitting the data. Because of $\mathbf{c}(t) \subset B$, we have the condition

$$
c_{1}(t)^{2}+c_{2}(t)^{2}+c_{3}(t)^{2}=1 .
$$

Thus, $\left(c_{1}, c_{2}, c_{3}\right)(t)$ is a curve on $S^{2}$. For interpolation purposes there exist nice algorithms for curve design on quadrics with rational curves, see [8]. Since there are no polynomial curves on $S^{2}$, the similar approximation algorithms with rational B-splines on the sphere become nonlinear. In addition, if the data points can be well approximated by a planar curve or a curve in a three-space, we have to take this into account here.

We do not want to go into details here but like to list two possibilities.

1. Apply a standard curve approximation with B-splines in the $l^{2}$-sense and project afterwards the solution curve to the Blaschke cylinder. If it is possible to choose a stereographic projection, the projected curve is a rational $\mathrm{B}$-spline, otherwise it is not rational.

2. Apply a biarc construction on $B$, which approximates the data points $b\left(T_{i}\right)$ by a sequence of ellipse arcs. The developable $D$ is thus approximated by a sequence of cones of rotation, see [10]. The biarc construction is a $G^{1}$-Hermite interpolation method, and it uses points $P_{j}$ plus tangent lines $V_{j}$ as input. To achieve this, we have to define the Hermite elements $P_{j}, V_{j}$ first. This can be done by approximating $b\left(T_{i}\right)$ with an arbitrary curve $\tilde{\mathbf{c}}(t)$ first, and $P_{j}, V_{j}$ are the evaluations of $\tilde{\mathbf{c}}(t)$ and $\dot{\tilde{\mathbf{c}}}(t)$ at chosen parameter values $t_{j}$. Any pair of Hermite elements $P_{j}, V_{j}$ and $P_{j+1}, V_{j+1}$ is interpolates by a pair of ellipse arcs on $B$. There exists a one- parameter family of solutions which can be parameterized over the projective line. But there is no guarantee that practicable solutions exist, with respect to the orientation given by the tangent vectors $V_{j}$, see [11].

Once having found a curve $\mathbf{c}(t)$ well approximating the image points $b\left(T_{i}\right)$, the one-parameter family $E(t)$ is already given by

$$
E(t): c_{4}(t)+c_{1}(t) x+c_{2}(t) y+c_{3}(t) z=0 .
$$

The envelope of $E(t)$ is the developable approximation $D^{*}$. Figure 7 shows an approximating curve to a set of image points $b\left(T_{i}\right)$.

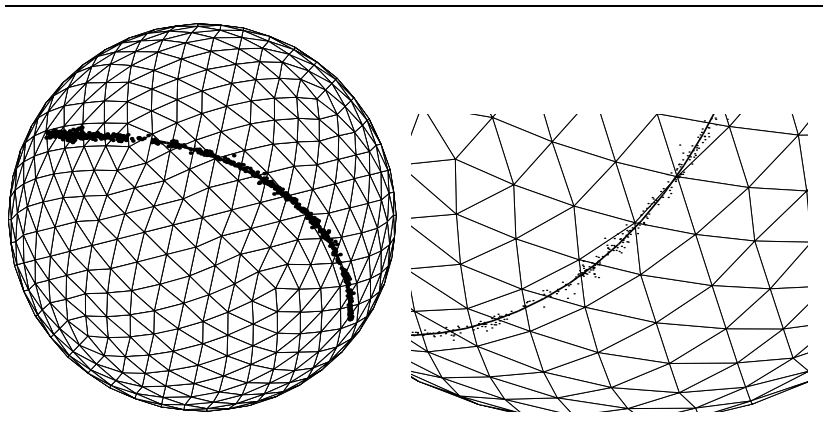

Figure 7. Approximating curve on $B$ with detail

We like to define the distance of the given surface $D$ and the approximation $D^{*}$. The approximation is a continuous model whereas $D$ is given by measurement points $\mathbf{p}_{i}$ and estimated tangent planes $T_{i}$ with associated parameter values $t_{i}$. If more emphasis is on the tangent planes, the squared distance of $D$ and $D^{*}$ can be defined by

$$
d^{2}\left(D, D^{*}\right)=\frac{1}{N} \sum_{i} d^{2}\left(T_{i}-E\left(t_{i}\right)\right),
$$

where the squared distances are computed according to (17). If more emphasis is on the measurements $\mathbf{p}_{i}$, the squared distance can be defined by

$$
d^{2}\left(D, D^{*}\right)=\frac{1}{N} \sum_{i} d^{2}\left(\mathbf{p}_{i}-E\left(t_{i}\right)\right)
$$

with respect to orthogonal distances between points $\mathbf{p}_{i}$ and planes $E\left(t_{i}\right)$.

\subsection{Boundary curves of the developable approxi- mation}

Once having computed the one-parameter family of planes $E(t)$ approximating the data points $\mathbf{p}_{i}$, we are able 
the generate a point representation of the developable approximation $D^{*}$. The generating lines $L(t)$ of $D^{*}$ are the intersection lines $E(t) \cap \dot{E}(t)$. We assume that there exist two bounding planes $H_{1}$ and $H_{2}$ of the domain of interest in a way that all generating lines $L(t)$ intersect $H_{1}$ and $H_{2}$ in proper points. The intersection curves of $L(t)$ and $H_{i}, i=1,2$ will be denoted by $\mathbf{c}_{i}(t)$ and are computed by

$$
\mathbf{c}_{i}(t)=E(t) \cap \dot{E}(t) \cap H_{i} .
$$

Thus, a point representation of $D^{*}$ is found by

$$
\mathbf{x}(t, u)=(1-u) \mathbf{c}_{1}(t)+u \mathbf{c}_{2}(t) .
$$

Figures 6 and 8 show developable surfaces approximating data points which are displayed as dots. The points displayed as stars are some singular points of the surface near the domain of interest.

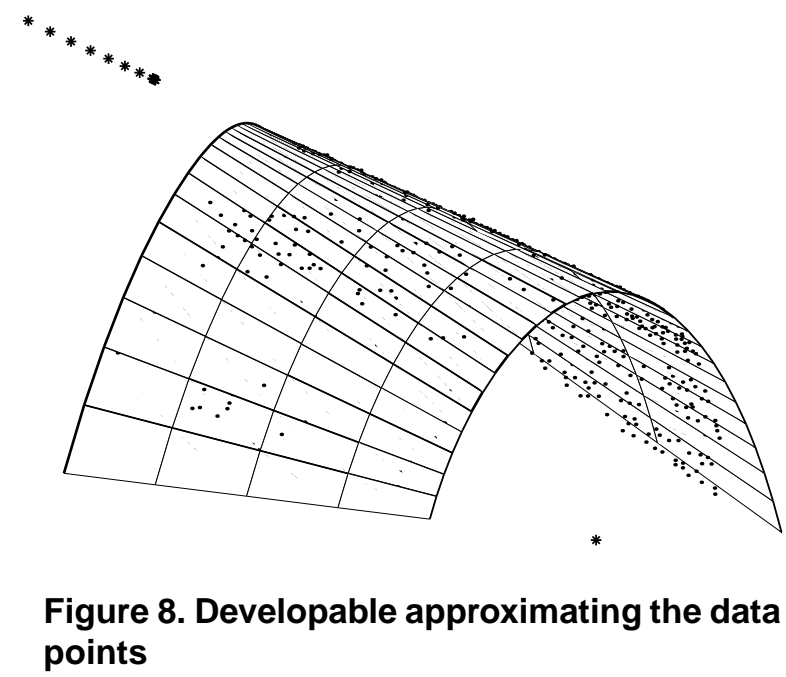

So far, we did not pay any attention to singular points of $D^{*}$. The control and avoidance of the singular points within the domain of interest is a complicated topic. We want to give some facts and ideas.

\subsection{Singular points of a developable surface}

In the case where the developable surface $D^{*}$ is given by a point representation, formula (4) describes the singular curve $\mathbf{s}(t)$. If $D^{*}$ is given by its tangent planes $E(t)$, the singular curve $\mathbf{s}(t)$ is the envelope of the lines $L(t)$ and thus computed by

$$
\mathbf{s}(t)=E(t) \cap \dot{E}(t) \cap \ddot{E}(t) .
$$

So we see that the singular curve $\mathbf{s}(t)$ depends in a highly nonlinear way on the coordinate functions of $E(t)$. On the Blaschke cylinder the situation is analogous. The singular point $\mathbf{s}$ of $E$ is in direct relation to the osculating plane

$$
\sigma=\mathbf{c}+\lambda \dot{\mathbf{c}}+\mu \ddot{\mathbf{c}}
$$

of the Blaschke image curve $b(E(t))=\mathbf{c}(t)$.

We compute the vector $\mathbf{n}=\mathbf{c} \wedge \dot{\mathbf{c}} \wedge \ddot{\mathbf{c}}$. The Cartesian coordinates of the singular curve are

$$
\mathbf{s}(t)=\frac{1}{n_{4}(t)}\left(n_{1}(t), n_{2}(t), n_{3}(t)\right)
$$

Zeros of the function $n_{4}$ are corresponding to points at infinity of $\mathbf{s}(t)$. If the data points $\mathbf{p}_{i}$ are contained in the unit sphere $\left(\left\|\mathbf{p}_{i}\right\| \leq 1\right)$, we require that $\mathbf{s}(t)$ satisfies

$$
\|\mathbf{s}(t)\|>1
$$

1. Assuming that $\mathbf{c}(t) \in B$ shall be represented by rational B-splines, the inequality (26) is a rather nasty side condition for the control points of $\mathbf{c}(t)$.

2. Let $\mathbf{c}(t) \in B$ be composed of biarcs. For two consecutive Hermite elements $P_{j}, V_{j}$ and $P_{j+1}, V_{j+1}$ there exists a one-parameter family of interpolating pairs of arcs. The condition (26) is a quadratic inequality in the free parameter. It restricts the family of practicable solutions which essentially depends on the choice of the Hermite elements, as we have mentioned in Section 5.2.

Pottmann and Wallner [15] have proposed a modelling scheme that uses a special representation

$$
E(t)=e_{4}(t)+e_{1}(t) x+t y-z=0,
$$

of the family of tangent planes $E(t)$ of $D^{*}$ but can handle the side condition (26) with relatively little costs. The parametrization implies that the planes $E(t)$ are graphs over the $x y$-plane and the generating lines $L(t)$ can never be parallel to the $y z$-plane. For appropriately chosen parts of the surface it is always possible to choose the coordinate system in a way that these properties hold. The choice of $e_{2}$ as parameter leads to a special parametric representation.

It would be possible to integrate this approach here. One has to partition the Blaschke image $b\left(T_{i}\right)$ in a way that for each part one can find a representation (27). The coordinate system has to be chosen in a way that the estimated planes $T_{i}$ are graphs ( $T_{i}$ are not parallel to the $z$-axis). This simplification has also be used in [13] for developable surface approximation. In addition it is necessary to rotate the coordinate system in a way that the considered part of the curvelike region $b\left(T_{i}\right)$ can be parameterized over the $y$-axis. 


\subsection{Correcting singular points}

Finally we want to give some hints to the treatment of singular points occurring in the approximation $D^{*}$. The singular points $\mathbf{S}$ correspond directly to the osculating planes of $\mathbf{c}$, according to (25). A good choice for $\mathbf{c}$ is an optimally stretched curve whose osculating planes do not vary much. If we choose B-Splines for approximation, the shape of a Bspline curve is strongly influenced by the choice of the knot sequence. Some experience and possibly user interaction seems to be necessary. If we choose the biarc-construction, the estimation and choice of the Hermite elements will have much influence on the final shape of $\mathbf{c}$ and $D^{*}$.

If singular points occur on the modelled surface patch we propose the following correction method. For that we assume that only a few singular points are present. The lines carrying these singular points shall not cover the whole surface.

By deleting the lines carrying singular points, we obtain a singularity-free surface with holes which are actually strips which have to be filled by regular developable pieces. We have to distinguish if the hole is bounded by two regular generating lines or if it occurs at the boundary.

1. The strip to be filled is bounded by two lines $L_{1}, L_{2}$ and tangent planes $T_{1}, T_{2}$ along them. These Hermite elements can be interpolated by a pair of cones of revolution (biarc-construction), chosen from a oneparameter family of solutions. As we have mentioned in Section 5.4, the set of practicable real solutions could be empty.

2. If the strip to be filled occurs at the boundary, we have to proceed in another way: Let $L_{1}$ be the 'last' regular generating line, with tangent plane $T_{1}$. We have to compute a regular developable, which approximates the data within the strip and which interpolates the Hermite element $L_{1}, T_{1}$. We can try to fit a single cone of revolution or a general cone whose vertex is outside the domain of interest. The cone of revolution can be computed as Blaschke image of a planar intersection of $B$ with a plane of regression to the Blaschke images of the data within the strip to be filled.

\subsection{Conclusion}

We have proposed a method for computing an approximating developable surface to measured data points. The approach uses estimated tangent planes and works in the Blaschke model $B$ of the space of planes. The reconstruction is performed by applying curve approximation with respect to a chosen metric on $B$. The control and correction of singularities of the approximating developable surface needs more research and detailed studies.

\section{References}

[1] R. Bodduluri and B. Ravani. Geometric design and fabrication of developable Bézier and B-spline surfaces. Transactions of the ASME, Journal of Mechanical Design, 116:1042-1048, 1994.

[2] J. Chalfant and T. Maekawa. Design for manufactoring using b-spline developable surfaces. Journal of Ship Research, 42, 3:207-215, 1998.

[3] H. Chen, I. Lee, S. Leopoldseder, H. Pottmann, T. Randrup, and J. Wallner. On surface approximation using developable surfaces. Graphical Models and Image Processing, 61:110 124, 1999.

[4] W. Frey and C. Wampler. Boundary triangulations approximating developable surfaces. Technical Report 8997, GM Research and Development Center, 1999.

[5] J. Hoschek. Dual bezier curves and surfaces. In R. Barnhill and W. Boehm, editors, Surfaces in Computer Aided Geometric Design, pages 147-156. North Holland, 1993.

[6] J. Hoschek and D. Lasser. Fundamentals of Computer Aided Geometric Design. AK Peters, Wellesley, MA, 1993.

[7] J. Hoschek and H. Pottmann. Interpolation and approximation with developable B-spline surfaces. In M. Daehlen, T. Lyche, and L. Schumaker, editors, Mathematical Methods for Curves and Surfaces, pages 255-264. Vanderbilt University Press, Nashville, TN, 1995.

[8] B. Jüttler and R. Dietz. A geometrical approach to interpolation on quadric surfaces. In P. Laurent, A. L. Méhauté, and L. Schumaker, editors, Curves and surfaces in geometric design, pages 251-258. AK Peters, 1994.

[9] I. Lee. Curve reconstruction from unorganized points. Computer Aided Geometric Design, 17:161-177, 2000.

[10] S. Leopoldseder and H. Pottmann. Approximation of developable surfaces with cone spline surfaces. Computer-Aided Design, 30:571-582, 1998.

[11] A. Nutbourne and R. Martin. Differential Geometry Applied to Curve and Surface Design, Vol.1, Foundations. Ellis Horwood, Chichester, 1988.

[12] F. Park, J. Yu, C. Chun, and B. Ravani. Design of developable surfaces using optimal control. Transactions of the ASME, Journal of Mechanical Design, 124:602-608, 2002.

[13] M. Peternell and H. Pottmann. Approximation in the space of planes - applications to geometric modeling and reverse engineering. RACSAM (Journal of the National Academy of Sciences of Spain, Series A, Mathematics), 96:243-256, 2002.

[14] H. Pottmann and G. Farin. Developable rational Bézier and B-spline surfaces. Computer Aided Geometric Design, 12:513-531, 1995.

[15] H. Pottmann and J. Wallner. Approximation algorithms for developable surfaces. Computer Aided Geometric Design, 16:539-556, 1999.

[16] H. Pottmann and J. Wallner. Computational Line Geometry. Springer, Berlin-Heidelberg-New York, 2001.

[17] T. Várády and R. Martin. Reverse engineering. In G. Farin, J. Hoschek, and M. Kim, editors, Handbook of Computer Aided Geometric Design, pages 651-681. Elsevier, 2002. 